\title{
CORRECCIÓN DE LA ACIDEZ DEL SUELO: EFECTOS EN EL CRECIMIENTO INICIAL DEL CAFÉ
}

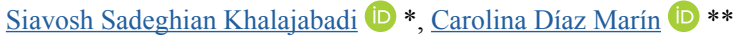

\begin{abstract}
Sadeghian-Khalajabadi, S., \& Díaz-Marín, C. (2020). Corrección de la acidez del suelo: Efectos en el crecimiento inicial del café. Revista Cenicafé, 71(1), 21-31. https://doi.org/10.38141/10778/1117

La acidez del suelo ha sido considerada una limitante común para la producción de café en la mayoría de las regiones de Colombia. Esta investigación tuvo como objetivo determinar el efecto de la corrección de la acidez del suelo en el crecimiento inicial de café (Coffea arabica L.). Para cinco suelos representativos de la zona cafetera de Colombia y con valores de $\mathrm{pH}<5,2$ se determinó el efecto de dosis crecientes de cal $\left(0,00 ; 1,25 ; 2,50 ; 5,00\right.$ y $\left.10,00 \mathrm{~g} \mathrm{dm}^{3}\right)$ en la biomasa seca total de las plantas, durante la fase de almácigo (seis meses). Con la aplicación de los tratamientos se logró corregir la acidez ( $\mathrm{pH}$ y $\mathrm{Al}^{3+}$ ) e incrementar los contenidos de $\mathrm{Ca}^{2+} \mathrm{y} \mathrm{Mg}^{2+}$ en todos los suelos estudiados, presentando algunas variaciones entre estos. En tres de los suelos se presentó efecto de los tratamientos en la materia seca de las plantas, respuesta que se relacionó principalmente con el aumento del $\mathrm{pH}$ y la neutralización del $\mathrm{Al}^{+3}$. El mayor crecimiento de las plantas (rendimientos relativos entre el 95 y 100\%) se asoció con los siguientes rangos de las propiedades e indicadores del suelo: $\mathrm{pH} 4,9-5,7, \mathrm{Ca}^{2+} 5,1-11,2 \mathrm{cmol}_{\mathrm{c}} \mathrm{kg}^{-1}, \mathrm{Mg}^{2+} 0,9-2,1 \mathrm{cmol}_{\mathrm{c}} \mathrm{kg}^{-1}$, saturación de bases 21-45\%, Ca:Mg 4,4:1-7,4:1, Ca:K 7:1-37:1, Mg:K 1,4:1-4,8:1, $\mathrm{Al}^{3+}<1,1 \mathrm{cmol}_{\mathrm{c}} \mathrm{kg}^{-1}$ y saturación de $\mathrm{Al}^{+3}<12 \%$.
\end{abstract}

Palabras clave: Coffea arabica, etapa de almácigo, encalado, $\mathrm{pH}$, aluminio, bases intercambiables.

\section{SOIL ACIDITY CORRECTION: EFFECTS ON INITIAL COFFEE GROWTH}

Soil acidity has been considered a common limiting factor for coffee production in most Colombian regions. The objective of this study was to determine the effect of correcting soil acidity in the initial stage of coffee growth (Coffea arabica L.).The effect of increasing lime doses (0.00, 1.25, 2.50, 5.00 and $10.00 \mathrm{~g}$ $\left.\mathrm{dm}^{3}\right)$ in the total dry biomass of plants during the nursery stage (6 months) was determined in five soils representative of the Colombian coffee zone and with $\mathrm{pH}$ values $<5.2$. The application of the treatments allowed to correct the acidity $\left(\mathrm{pH}\right.$ and $\mathrm{Al}^{3+}$ ) and to increase the contents of $\mathrm{Ca}^{2+}$ and $\mathrm{Mg}^{2+}$ in all the studied soils, with some variations among them. Three of the soils showed an effect of the treatments on the dry matter of the plants, a response mainly related to the increase in $\mathrm{pH}$ and the neutralization of $\mathrm{Al}^{+3}$. The higher growth of the plants (relative yields between 95 and 100\%) was associated with the following ranges of soil properties and indicators: $\mathrm{pH} 4.9-5.7, \mathrm{Ca}^{2+} 5.1-11.2 \mathrm{cmol}_{\mathrm{c}} \mathrm{kg}^{-1}, \mathrm{Mg}^{2+} 0.9-2.1 \mathrm{cmol}_{\mathrm{c}} \mathrm{kg}^{-1}$, base saturation 21\%-45\%, Ca:Mg 4.4:1-7.4:1, Ca:K 7:1-37:1, Mg:K 1.4:1-4.8:1, $\mathrm{Al}^{3+}<1.1 \mathrm{cmol}_{\mathrm{c}} \mathrm{kg}^{-1}$ and saturation of $\mathrm{Al}^{+3}<12 \%$.

Keywords: Coffea arabica, seedling stage, liming, pH, aluminum, exchangeable bases.

* Investigador Científico III. Disciplina de Suelos, Centro Nacional de Investigaciones de Café, Cenicafé. Chinchiná, Caldas, Colombia. https://orcid.org/0000-0003-1266-0885.

** Ingeniera agrónoma, M.Sc. https://orcid.org/0000-0002-6843-7503. 
Entre las limitantes más comunes para la producción de café en Colombia se encuentra la acidez del suelo, siendo la situación más crítica en departamentos como Antioquia, Cundinamarca y Santander (Sadeghian, 2013). En los suelos ácidos las concentraciones altas de aluminio $\left(\mathrm{Al}^{3+}\right)$ y manganeso $\left(\mathrm{Mn}^{2+}\right)$ solubles resultan tóxicas para las plantas; además, se reduce la disponibilidad de algunos nutrientes, principalmente calcio $\left(\mathrm{Ca}^{2+}\right)$, magnesio $\left(\mathrm{Mg}^{2+}\right)$, fósforo y nitrógeno (Havlin et al., 2014; Raij, 2011).

La alternativa más común para la corrección de la acidez resulta del uso de enmiendas de reacción básica, particularmente carbonatos de calcio y/o magnesio; práctica que se denomina encalado o encalamiento. El ejercicio del encalado en café (Coffea arabica L.) favorece el desarrollo del cultivo, tanto para el crecimiento inicial (Pavan et al., 1982; Rodrigues et al., 2001; Rodrigues et al., 2006) como en la fase productiva (Chaves et al., 1984; Corrêa et al., 2007).

Pese a que en Colombia muchas de las áreas dedicadas al cultivo de café presentan problemas de acidez, son contadas las investigaciones en torno a su manejo; al respecto, puede mencionarse el trabajo realizado por Suárez y Rodríguez (1956) para cafetales jóvenes y el experimento conducido por Valencia y Bravo (1981) para plantaciones en la fase reproductiva. Con respecto a la etapa de almácigo, se cuenta con la información generada en una investigación realizada por Toro (1994). Cabe resaltar que los resultados obtenidos en los trabajos en mención no fueron concluyentes, pues no revelaron efectos contundentes del encalado en las variables evaluadas (producción o biomasa de la planta).

Por otro lado, diferentes métodos e indicadores han sido propuestos para determinar los requerimientos de la cal; entre los más comunes pueden citarse: el contenido de $\mathrm{Al}^{3+}$ y el respectivo porcentaje de saturación con respecto a Capacidad de Intercambio Catiónico Efectiva-CICE (Kamprath, 1970), el porcentaje de saturación de bases (Raij et al., 1983), la neutralización del $\mathrm{Al}^{3+}$ y el aumento de los tenores de $\mathrm{Ca}^{2+}$ y $\mathrm{Mg}^{2+}$ intercambiables (Álvarez \& Ribeiro, 1999), el uso de una solución tamponada con el fin de estimar los requerimientos de cal para alcanzar un nivel requerido de $\mathrm{pH}$ (Shoemaker et al., 1961) y la incubación. Para condiciones de Colombia se sugiere tener en cuenta el $\mathrm{pH}$ y el contenido de $\mathrm{Ca}^{2+}$ (Sadeghian, 2008); en este sentido, los valores que se consideran adecuados para pH están comprendidos entre 5,0 y 5,5, y los contenidos de $\mathrm{Ca}^{2+}$ mayores de $3,0 \mathrm{cmol}_{\mathrm{c}} \mathrm{kg}^{-1}$.

Dado que en el ámbito nacional se cuenta con poca información acerca del tema, en este trabajo se planteó como objetivo, determinar el efecto de la corrección de la acidez del suelo en el crecimiento inicial de café.

\section{MATERIALES Y MÉTODOS}

La fase experimental de la investigación se desarrolló en el Centro Nacional de Investigaciones de Café, Cenicafé, ubicado en el municipio de Manizales, departamento de Caldas, a $5^{\circ} 1^{\prime}$ latitud Norte y $75^{\circ} 35^{\prime}$ longitud Oeste, con $1.310 \mathrm{~m}$ de altitud, temperatura promedio de $21,7^{\circ} \mathrm{C}$ y precipitación anual de $2.600 \mathrm{~mm}$.

En cuatro Estaciones Experimentales de Cenicafé y una finca cafetera se recolectaron muestras de suelo a $20 \mathrm{~cm}$ de profundidad, en cafetales que no habían sido encalados durante los últimos cinco años. La selección de los sitios se basó en su acidez $(\mathrm{pH}<5,2)$ $\mathrm{y}$ el contraste en las propiedades químicas y físicas (Tabla 1). Una vez homogeneizados los suelos mediante volteos y eliminados los terrones grandes con el uso de zaranda, 
de cada uno, se apartaron 150 decímetros cúbicos $\left(\mathrm{dm}^{3}\right)$; cantidad que fue dividida en cinco porciones de $30 \mathrm{dm}^{3}$ y tratada cada una con las siguientes dosis de cal $(46,6 \%$ de $\mathrm{CaO}$ y $8,1 \%$ de $\mathrm{MgO}$ ), aplicadas como tratamientos: 0,$00 ; 1,25 ; 2,50 ; 5,00$ y 10,00 $\mathrm{g} \mathrm{dm}^{3}$. Posteriormente, los suelos recibieron riego hasta alcanzar humedades cercanas a la capacidad de campo (agua retenida a presiones entre 0,033 y $1,5 \mathrm{MPa}$ ) y se incubaron por 30 días (tiempo definido en ensayos previos como suficiente para que reaccionara la mayor parte de la enmienda); por último, se tomó una muestra para el análisis de las propiedades químicas, evaluadas según los procedimientos descritos por Carrillo (1985). Con el propósito de determinar el efecto de los tratamientos en la disponibilidad del nitrógeno nítrico- $\mathrm{NO}^{3-}$, se valoró esta fracción mediante el método RQ-Flex.

Con el suelo de cada localidad y tratamiento se llenaron 15 bolsas de polietileno negro, con una capacidad de $2,0 \mathrm{dm}^{3}$, para luego sembrar en ellas plántulas de café variedad Colombia (una por bolsa). Las 75 unidades experimentales de cada suelo, resultantes de cinco tratamientos (dosis de enmienda) x 15 repeticiones de cada suelo, fueron dispuestas bajo el diseño estadístico completamente aleatorio en un umbráculo. Para el manejo agronómico, se tuvieron en cuenta las recomendaciones de la Federación Nacional de Cafeteros de Colombia-FNC (2013) en lo concerniente al manejo de arvenses, riego, sombra y manejo fitosanitario. Después de seis meses de la siembra, se extrajeron del suelo las raíces, se secaron las plantas durante $72 \mathrm{~h}$ en estufa, a $65^{\circ} \mathrm{C}$, y se determinó el peso de la materia seca total de cada una.

Se realizó un análisis de varianza por localidad ( $\alpha=95 \%$ ) con el fin de determinar el efecto de los tratamientos en la biomasa seca total de las plantas. Mediante el uso de regresiones se seleccionaron modelos para explicar el efecto de las dosis de cal en las variables de respuesta (planta y suelo). Los datos fueron analizados mediante el programa estadístico SAS (Statistical Analysis System), versión 9,2 de 2009.

Cuando se presentó efecto de los tratamientos en la biomasa de las plantas, se calcularon las dosis de cal requeridas para alcanzar el máximo rendimiento (100\%) y el $95 \%$ de este, de acuerdo al modelo del mejor ajuste. Posteriormente, se determinaron para este rango los valores correspondientes de $\mathrm{pH}, \mathrm{Al}^{3+}, \mathrm{Ca}^{2+}, \mathrm{Mg}^{2+}$, saturación de $\mathrm{Al}^{3+}$, saturación de bases intercambiables y las relaciones $\mathrm{Ca}: \mathrm{Mg}, \mathrm{Ca}: \mathrm{K}$ y $\mathrm{Mg}: \mathrm{K}$. En este caso, el valor correspondiente al $95 \%$ se empleó para calcular el nivel crítico de las propiedades en mención y el intervalo entre el $95 \%$ y $100 \%$ como el rango adecuado.

\section{RESULTADOS Y DISCUSIÓN}

La aplicación de las dosis crecientes del material encalante incrementó el $\mathrm{pH}, \mathrm{Ca}^{2+} \mathrm{y}$ $\mathrm{Mg}^{2+}$ en todos los suelos, mientras que el $\mathrm{Al}^{3+}$ disminuyó significativamente para los suelos de Jamundí y El Rosario, donde los contenidos de este elemento eran más altos (Tabla 2). Dado que los tratamientos afectaron de manera diferencial a los suelos en sus contenidos de las bases intercambiables, las relaciones $\mathrm{Ca} / \mathrm{Mg}$, $\mathrm{Ca} / \mathrm{K}$ y $\mathrm{Mg} / \mathrm{K}$ también presentaron variaciones considerables (Figura 1).

Los tratamientos tuvieron efectos significativos en la biomasa seca total de las plantas desarrolladas en los suelos de Jamundí, El Rosario y Paraguaicito (Figura 2). Con respecto a las primeras dos localidades, la respuesta puede relacionarse con un mayor control de la acidez, debido a sus valores más bajos de $\mathrm{pH}(4,5$ y 4,4 , respectivamente) y contenidos elevados de $\mathrm{Al}^{3+}$ (en su orden 


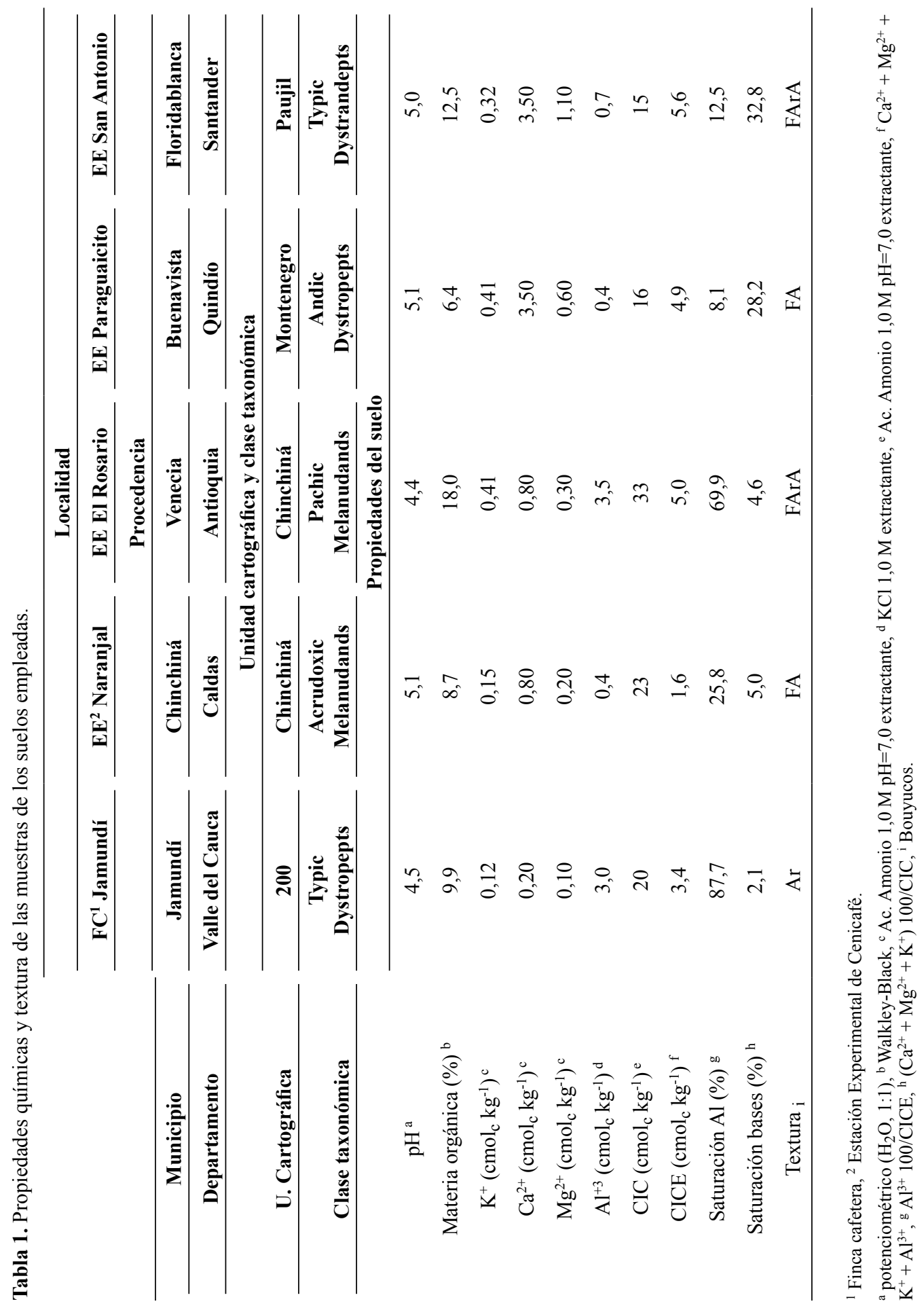


Tabla 2. Ecuaciones de regresión para $\mathrm{pH}$, calcio, magnesio y aluminio en función de las dosis de cal suministradas en los suelos evaluados.

\begin{tabular}{|c|c|c|}
\hline Localidad & Ecuación de regresión & $\mathbf{R}^{2}$ \\
\hline & pH & \\
\hline FC Jamundí & $\hat{\mathrm{y}}=4,5275^{* *}+0,2060^{* *} \mathrm{x}$ & 0,999 \\
\hline EE Naranjal & $\hat{\mathrm{y}}=5,1151^{* *}+2,0744^{* *}\left(1-\mathrm{e}^{\left(-0,1954^{* *} \mathrm{x}\right)}\right)$ & 0,999 \\
\hline EE Paraguaicito & $\hat{\mathrm{y}}=5,1275^{* *}+0,1100^{* *} \mathrm{x}$ & 0,980 \\
\hline EE El Rosario & $\hat{\mathrm{y}}=4,4175^{* *}+0,1180^{* *} \mathrm{x}$ & 0,998 \\
\hline \multirow[t]{2}{*}{ EE San Antonio } & $\hat{\mathrm{y}}=4,9888^{* *}+1,8918^{* *}\left(1-\mathrm{e}^{\left(-0,2480^{* *} \mathrm{x}\right)}\right)$ & 0,995 \\
\hline & Calcio-Ca ${ }^{2+}\left(\mathrm{cmol}_{\mathbf{c}} \mathrm{kg}^{-1}\right)$ & \\
\hline FC Jamundí & $\hat{\mathrm{y}}=0,4825^{* *}+1,3060 * * \mathrm{x}$ & 0,997 \\
\hline EE Naranjal & $\hat{\mathrm{y}}=1,5625^{*}+1,1140^{* *} \mathrm{x}$ & 0,973 \\
\hline EE Paraguaicito & $\hat{y}=3,6523 *+1,18280 * * x-0,0522 * x^{2}$ & 0,993 \\
\hline EE El Rosario & $\hat{\mathrm{y}}=0,7225^{*}+1,1460^{* *} \mathrm{x}$ & 0,996 \\
\hline \multirow[t]{2}{*}{ EE San Antonio } & $\left.\hat{y}=3,2231 *+2,1721 * x-0,1065^{*} x^{2}\right)$ & 0,987 \\
\hline & Magnesio- $\mathrm{Mg}^{2+}\left(\mathrm{cmol}_{\mathrm{c}} \mathrm{kg}^{-1}\right)$ & \\
\hline FC Jamundí & $\hat{\mathrm{y}}=0,1369+0,3012 * * \mathrm{x}-0,0236^{*} \mathrm{x}^{2}$ & 0,983 \\
\hline EE Naranjal & $\hat{\mathrm{y}}=1,2636^{* *}-1,0748^{* *} \mathrm{e}^{(-0,3889 * \mathrm{x})}$ & 0,991 \\
\hline EE Paraguaicito & $\hat{y}=1,2569 * *-0,6475^{* *} \mathrm{e}^{(-0,4626 x)}$ & 0,976 \\
\hline EE El Rosario & $\hat{\mathrm{y}}=0,3975^{* *}+0,1820^{* *} \mathrm{x}$ & 0,992 \\
\hline \multirow[t]{2}{*}{ EE San Antonio } & $\hat{\mathrm{y}}=1,1000^{* *}+0,2369^{* *} \mathrm{x}-0,0206^{* *} \mathrm{x}^{2}$ & 0,975 \\
\hline & Aluminio-Al ${ }^{3+}\left(\mathrm{cmol}_{\mathbf{c}} \mathbf{~ k g}^{-1}\right)$ & \\
\hline FC Jamundí & $\hat{\mathrm{y}}=2,999 * * \mathrm{e}^{\left(-0,6763^{* *} \mathrm{x}\right)}$ & 0,996 \\
\hline EE Naranjal & $\hat{\mathrm{y}}=\overline{\mathrm{Y}}=0,16 \mathrm{~ns}$ & \\
\hline EE Paraguaicito & $\hat{\mathrm{y}}=\overline{\mathrm{Y}}=0,18 \mathrm{~ns}$ & \\
\hline EE El Rosario & $\hat{y}=3,4454 * * e^{(-0,2762 * * x)}$ & 0,996 \\
\hline EE San Antonio & $\hat{\mathrm{y}}=\overline{\mathrm{Y}}=0,28 \mathrm{~ns}$ & \\
\hline
\end{tabular}

*significancia al 5\%; **1\% de probabilidad; ns no significativo.

3,0 y $\left.3,5 \mathrm{cmol}_{\mathrm{c}} \mathrm{kg}^{-1}\right)$. Reportes similares han sido entregados por Pavan y Bingham (1982), Pavan et al. (1982), Rodrigues et al. (2001) y Rodrigues et al. (2006), para el crecimiento inicial de café. Con respecto al suelo de Paraguaicito, el efecto de los tratamientos no guarda una relación estrecha con los componentes de la acidez o las bases intercambiables (Tabla 1), ya que en general sus niveles se encontraban dentro de los rangos que se consideran óptimos, según lo definido por Sadeghian (2013). Una posible causa estaría asociada con el aumento considerable del nitrógeno nítrico generado por el incremento del $\mathrm{pH}$, cuya magnitud fue mayor que las demás localidades (Figura 3).

Aunque el encalamiento no tuvo efectos estadísticamente significativos en el crecimiento de las plantas desarrolladas en los suelos de Naranjal y Santander, se observaron ligeros incrementos al aplicar dosis bajas de cal $\left(1,25\right.$ y $\left.2,5 \mathrm{~g} \mathrm{dm}^{3}\right)$. Para Naranjal la respuesta observada podría estar relacionada con los contenidos bajos de las bases intercambiables (Tabla 1). De acuerdo con Álvarez y Ribeiro (1999) y Sadeghian (2008), los requerimientos en la cantidad 
de la cal, además de la acidez del suelo también dependen de los contenidos de $\mathrm{Ca}^{2+}$ y $\mathrm{Mg}^{2+}$, sugerencia enfocada a una mayor sustentabilidad de los tenores de estos dos elementos en los suelos. En cuanto al suelo de la EE San Antonio, la tendencia registrada estaría relacionada con la posible neutralización de los altos contenidos de hierro y parcialmente manganeso (Figura 4). Se hubiera esperado disminución en el crecimiento de las plantas al aplicar la dosis más elevada $\left(10,0 \mathrm{~g} \mathrm{dm}^{3}\right)$, particularmente en aquellos suelos donde el pH alcanzó valores superiores a 6,0 (Jamundí, Santander y Naranjal); sin embargo, sólo se observó este comportamiento para Jamundí.
Los valores máximos de la biomasa seca total (Rendimiento Relativo-RR=100\%), estimados para Jamundí, Paraguaicito y El Rosario fueron de 3,48, 4,42 y 4,39 g/planta, respectivamente. Con base en esta información se calcularon los niveles críticos $(\mathrm{RR}=95 \%)$ y los rangos óptimos (RR entre 95\% y 100\%) de las propiedades del suelo relacionadas con la acidez y el encalamiento (Tabla 2). Los rangos óptimos estuvieron entre los siguientes valores: $\mathrm{pH} 4,9$ y 5,7, Ca 5,1 y $11,2 \mathrm{cmol}_{\mathrm{c}}$ $\mathrm{kg}^{-1}, \mathrm{Mg} 0,9$ y 2,1 $\mathrm{cmol}_{\mathrm{c}} \mathrm{kg}^{-1}$, saturación de bases 21 y $45 \%$, relación Ca: $\mathrm{Mg} 4,4: 1$ y 7,4:1, relación $\mathrm{Ca}: \mathrm{K}$ 7:1 y 37:1 y relación $\mathrm{Mg}: \mathrm{K}$ 1,4:1 y 4,8:1. Los niveles críticos de $\mathrm{Al}^{3+}$

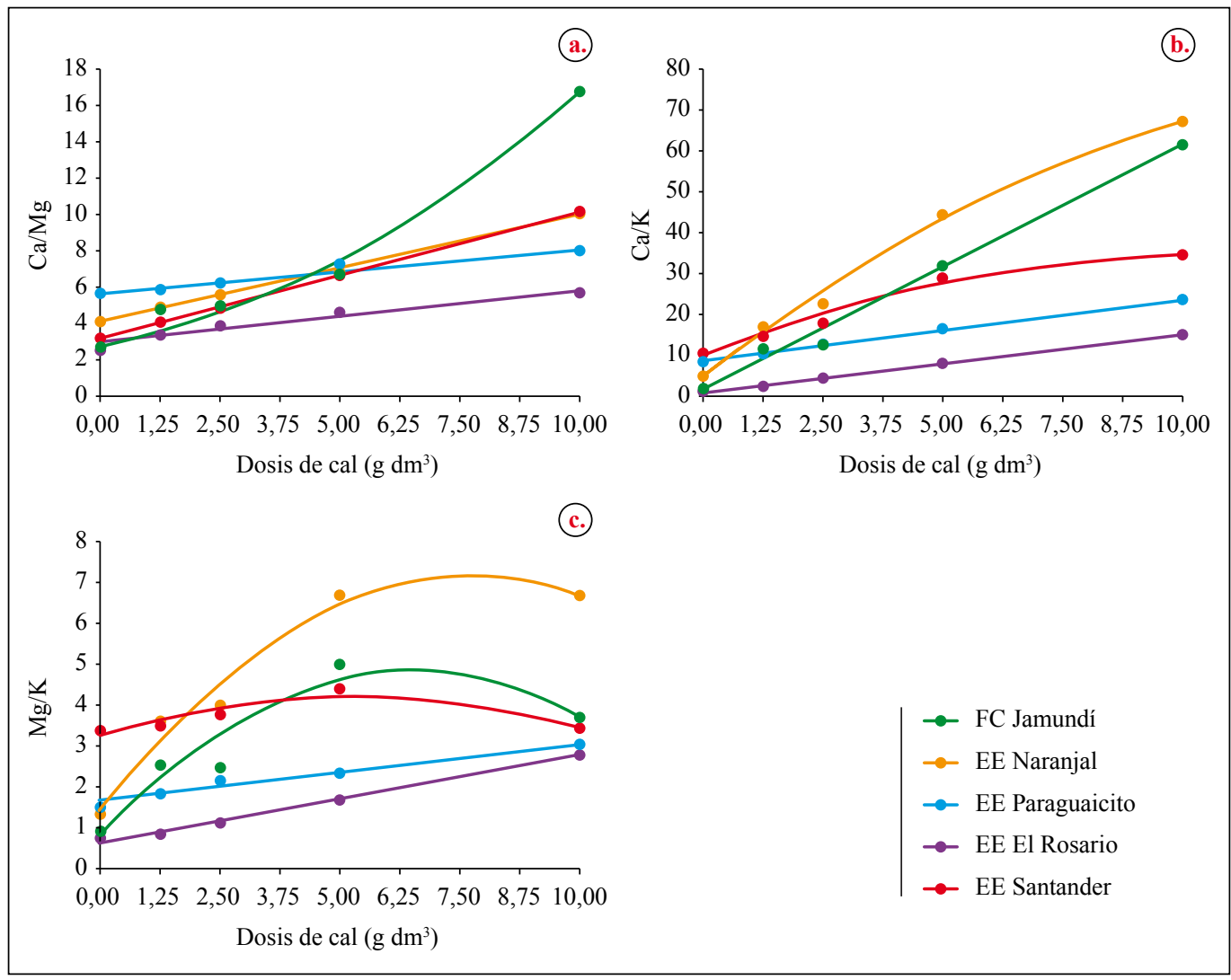

Figura 1. Cambios en las relaciones $\mathrm{Ca} / \mathrm{Mg}(\mathrm{a}), \mathrm{Ca} / \mathrm{K}$ (b) y $\mathrm{Mg} / \mathrm{K}$ (c) intercambiables en respuesta a las dosis del material encalante empleado. 

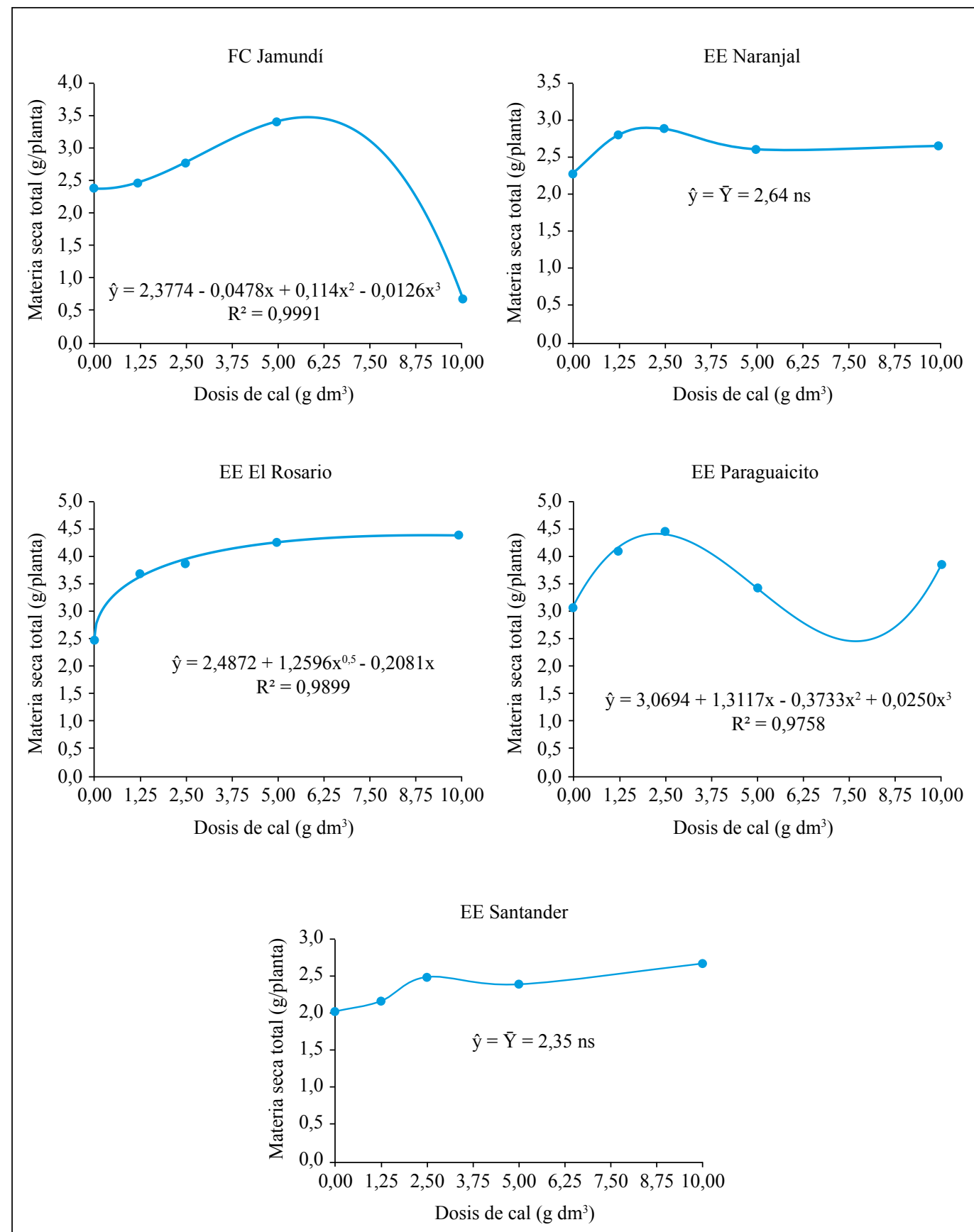

Figura 2. Materia seca total de las plantas de café en respuesta a las dosis de cal, aplicadas en cinco suelos de la zona cafetera. 


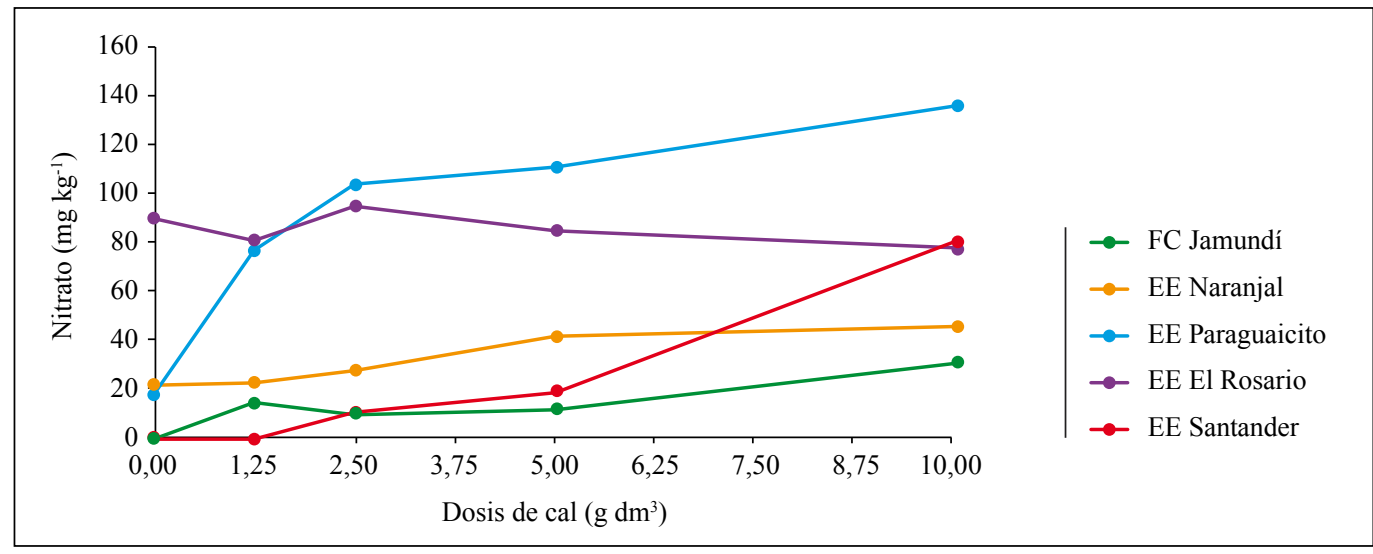

Figura 3. Variaciones en los contenidos de nitrato en respuesta a las dosis de cal.

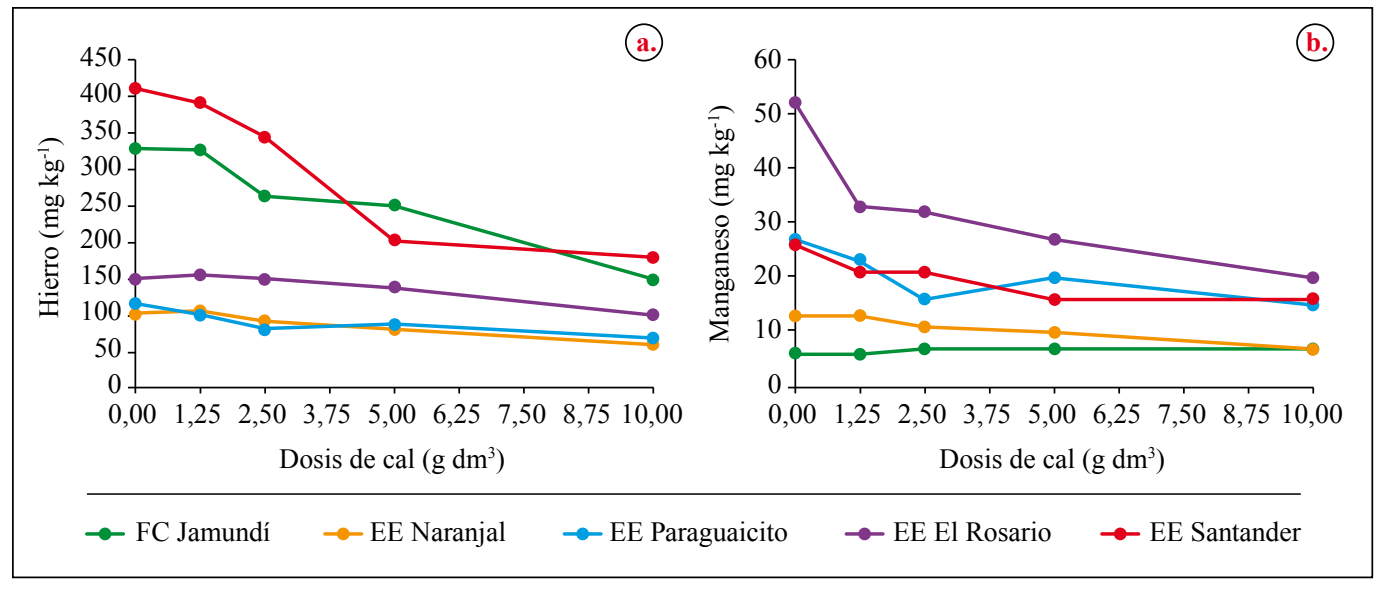

Figura 4. Variaciones en los contenidos de hierro (a) y manganeso (b) del suelo en respuesta a las dosis de cal.

y saturación de $\mathrm{Al}^{+3}$ fueron menores de 1,1 $\mathrm{cmol}_{\mathrm{c}} \mathrm{kg}^{-1}$ y $12 \%$, respectivamente.

Con respecto al $\mathrm{pH}$ y $\mathrm{Al}^{3+}$, los hallazgos de este estudio confirman los rangos sugeridos por Sadeghian $(2008 ; 2013)$ para condiciones de Colombia y, en cuanto al $\mathrm{Ca}^{+2} \mathrm{y} \mathrm{Mg}^{+2}$, sus niveles son parcialmente mayores a los reportados como óptimos (mayor de 3,0 y $0,9 \mathrm{cmol}_{\mathrm{c}} \mathrm{kg}^{-1}$, respectivamente), ello como consecuencia de la baja solubilidad de la enmienda aplicada. Los rangos para $\mathrm{Al}^{3+}$ intercambiable y saturación de $\mathrm{Al}^{3+}$ coinciden de alguna manera con los niveles críticos hallados por Pavan et al. (1982) para el crecimiento de las raíces de café $\left(0,3\right.$ a $1,3 \mathrm{cmol}_{\mathrm{c}} \mathrm{kg}^{-1} \mathrm{y}$ $3 \%$ a $25 \%$, respectivamente). Los valores del porcentaje de saturación de bases $(\% \mathrm{SB})$ son menores a los indicados por otros investigadores para el cultivo de café, es decir, $60 \%$ a $70 \%$ (Álvarez \& Ribeiro, 1999; Corrêa et al., 2007; Raij et al., 1983), resultado que puede atribuirse a la elevada CIC de los suelos en este estudio, relacionado con su riqueza en los contenidos 
de la MO. De acuerdo con Guarçoni (2017) el valor sugerido del $\%$ SB para café puede variar según la CIC, determinada en el laboratorio a $\mathrm{pH}$ 7,0; al respecto, este autor propone los siguientes porcentajes: $90 \%$ para CIC baja (menor de 4,30 $\mathrm{cmol}_{\mathrm{c}} \mathrm{dm}^{3}$ ), 70\% para $\mathrm{CIC}$ media (entre 4,30 y $8,60 \mathrm{cmol}_{\mathrm{c}} \mathrm{dm}^{3}$ ) y $60 \%$ para CIC alta (mayor de 15,0 $\mathrm{cmol}_{\mathrm{c}} \mathrm{dm}^{3}$ ). Los anteriores valores siguen siendo altos para los resultados obtenidos en este estudio, en especial para el suelo de la EE El Rosario con una $\mathrm{CIC}=33 \mathrm{cmol}_{\mathrm{c}} \mathrm{kg}^{-1}$, indicando de esta manera que el \%SB puede no ser un buen indicador para los suelos de Colombia con altos niveles de MO.

En cuanto a las relaciones entre las bases intercambiables, Bear y Toth (1948) sugieren los siguientes valores para $\mathrm{Ca}-\mathrm{Mg}, \mathrm{Ca}-\mathrm{K}$ y Mg-K: 6,5:1, 13,0:1 y 2,0:1, respectivamente. Para Malavolta (2006) una relación de Ca:Mg hasta 4,5:1, favorece la producción de café, no así para relaciones mayores a 5:1; a su vez, García (1981), verificó que las relaciones 2,52:1 y 3,08:1 fueron las más favorables para el desarrollo de las plantas café en dos suelos diferentes. Los resultados obtenidos en esta investigación demuestran que estas relaciones varían ampliamente según el tipo de suelo y, por lo tanto, no deben considerarse con criterios fijos.

Los resultados del presente estudio permiten concluir que:

- La corrección de la acidez del suelo contribuye al crecimiento del café durante la etapa inicial del cultivo, principalmente cuando el $\mathrm{pH}$ del suelo es menor de 5,0 y el aluminio intercambiable $\left(\mathrm{Al}^{3+}\right)$ mayor de $1,0 \mathrm{cmol}_{\mathrm{c}} \mathrm{kg}^{-1}$.

- El mayor crecimiento de las plantas se logra con valores de $\mathrm{pH}$ entre 4,9 y 5,7.

Tabla 3. Dosis de cal y propiedades del suelo, estimados para tres suelos evaluados y rendimientos relativos (RR) de 95 y $100 \%$.

\begin{tabular}{|c|c|c|c|c|c|c|}
\hline \multirow{2}{*}{$\begin{array}{c}\text { Dosis requerida y } \\
\text { propiedades del suelo }\end{array}$} & \multicolumn{2}{|c|}{ FC Jamundí } & \multicolumn{2}{|c|}{ EE Paraguaicito } & \multicolumn{2}{|c|}{ EE EI Rosario } \\
\hline & $\mathrm{RR}=95 \%$ & $\mathrm{RR}=100 \%$ & $\mathrm{RR}=95 \%$ & $\mathrm{RR}=100 \%$ & $\mathrm{RR}=95 \%$ & $\mathrm{RR}=100 \%$ \\
\hline Dosis de cal $\left(\mathrm{g} \mathrm{dm}^{3}\right)$ & 4,41 & 5,81 & 1,30 & 2,28 & 4,15 & 9,15 \\
\hline $\mathrm{pH}$ & 5,44 & 5,72 & 5,27 & 5,38 & 4,91 & 5,50 \\
\hline $\mathrm{Al}^{3+}\left(\mathrm{cmol}_{\mathrm{c}} \mathrm{kg}^{-1}\right)$ & 0,15 & 0,06 & 0,22 & 0,15 & 1,10 & 0,28 \\
\hline $\mathrm{Ca}^{2+}\left(\mathrm{cmol}_{\mathrm{c}} \mathrm{kg}^{-1}\right)$ & 6,24 & 8,07 & 5,10 & 6,08 & 5,48 & 11,21 \\
\hline $\mathrm{Mg}^{2+}\left(\mathrm{cmol}_{\mathrm{c}} \mathrm{kg}^{-1}\right)$ & 1,01 & 1,09 & 0,90 & 1,03 & 1,15 & 2,06 \\
\hline Saturación de Al (\%) & 1,81 & 0,53 & 3,06 & 1,47 & 11,84 & 1,43 \\
\hline Saturación de bases (\%) & 33,97 & 43,09 & 38,65 & 45,32 & 20,59 & 39,40 \\
\hline Relación Ca:Mg & $6,20: 1$ & $7,40: 1$ & $5,66: 1$ & $4,37: 1$ & $4,75: 1$ & $5,43: 1$ \\
\hline Relación Ca:K & $28,35: 1$ & $36,77: 1$ & 10,93:1 & $12,39: 1$ & $7,07: 1$ & $14,20: 1$ \\
\hline Relación $\mathrm{Mg}: \mathrm{K}$ & $4,41: 1$ & $4,79: 1$ & $1,83: 1$ & $2,03: 1$ & $1,42: 1$ & $2,60: 1$ \\
\hline
\end{tabular}


- Para el crecimiento inicial del café, los rangos óptimos de calcio, magnesio, saturación de $\mathrm{Al}^{3+}$, saturación de bases y las relaciones entre las bases intercambiables, pueden presentar diferencias, tratándose de un suelo y otro.

\section{LITERATURA CITADA}

Álvarez, V., \& Ribeiro, A. (1999). Calagem. En A. Ribeiro, P. Tacitó \& V. Álvarez (Eds.). Recomendação para o uso de corretivos e fertilizantes em Minas Gerais: 5 a aproximação (pp. 43-60). Comissão de Fertilidade do Solo do Estado de Minas Gerais.

Bear, F.E., \& Toth, S.J. (1948). Influence of Calcium on availability of other soil cations. Soil Science, 65(1), 69-74. https://journals.lww.com/soilsci/ citation/1948/01000/influence_of_calcium_on_ availability_of_other_soil.7.aspx

Carrillo, I. F. (1985). Manual de laboratorio de suelos. Cenicafé.

Chaves, J., Pavan, M.A., \& Igue, K. (1984). Respostas do cafeeiro à calagem. Pesquisa Agropecuária Brasileira, 19(5), 573-582. https://seer.sct.embrapa.br/index.php/ pab/article/view/15714/9728

Corrêa, J.B., Reis, T.H., Pozza, A., Guimarães, P.T., \& Carvalho, J. (2007). Índice de saturação por bases na nutrição e na produtividade de cafeeiros Catuaí vermelho (Coffea arabica L.). Coffee Science, 2(2), 159-167. https://pdfs.semanticscholar.org/552a/13ccca2a5a05a 36e5f83a9ff4f2ed1ff1a10.pdf

Federación Nacional de Cafeteros de Colombia. (2013). Manual del cafetero colombiano: Investigación y tecnología para la sostenibilidad de la caficultura (Vol. 3). Cenicafé.

Garcia,A. (1981). Calagem para o cafeeiro. En E. Malavolta, T. Yamada \& J. Guidolin(Eds.), Nutrição e adubação do cafeeiro (pp. 103-115). Instituto da Potassa \& Fosfato e Instituto Internacional da Potassa.

Guarçoni, A. (2017). Saturação por bases para o cafeeiro baseada no $\mathrm{pH}$ do solo e no suprimento de $\mathrm{Ca}$ e $\mathrm{Mg}$. Coffee Science, 12(3), 327-336. http://hdl.handle. net/123456789/9123

Havlin, J.L., Tisdale, S.L., Nelson, W.L., \& Beaton, J.D. (2014). Soil fertility and fertilizers: An introduction to nutrient management (8 ed.). Pearson.
Kamprath, E. J. (1970). Exchangeable aluminum as a criterion for liming leached mineral soils. Soil Science Society of America Journal, 34(2), 252-254. https:// doi.org/10.2136/sssaj1970.03615995003400020022x

Malavolta, E. (2006). Manual de nutrição mineral de plantas. Agronômica Ceres.

Pavan, M.A., \& Bingham, F.T.(1982). Toxicity of aluminum to coffee seedlings grown in nutrient solution. Soil Science Society of America Journal, 46(5), 993-997. https:// doi.org/10.2136/sssaj1982.03615995004600050021x

Pavan, M.A., Bingham, F.T., \& Pratt, P.F. (1982). Toxicity of aluminum to coffee in Ultisols and Oxisols amended with $\mathrm{CaCO}_{3}, \mathrm{MgCO}_{3}$ and $\mathrm{CaSO}_{4} \cdot 2 \mathrm{H}_{2} \mathrm{O}$. Soil Science Society of America Journal, 46(6), 1201-1207. https://doi.org/10.2136/ sssaj1982.03615995004600060017x

Raij, B. (2011). Fertilidade do solo e manejo de nutrientes. International Plant Nutrition Institute.

Raij, B., Pereira, A., Cantarella, H., \& Machado, N., (1983). Alumínio trocável e saturação em bases como critérios para recomendação de calagem. Bragantia, 42(1), 149-156. http://www.scielo.br/pdf/brag/v42n1/13.pdf

Rodrigues, L.A., Martinez, H.E.P., Neves, J.C., Novais, R.F., \& Mendonça, S.M (2001). Growth response of coffee tree shoots and roots to subsurface liming. Plant and Soil, 234(2), 207-214. https://doi. org/10.1023/A:1017999318532

Rodrigues, L.A., Matrines, H.E., Lima, J.C., Novais, R., \& Mendonça, S. M. (2006). Respostas nutricionais de cafeeiros Catuaí e Icatu a doses de calcário em subsuperfície. Revista Brasileira de Ciência do Solo, 30(6), 985-995. https://doi.org/10.1590/S010006832006000600008

Sadeghian, S. (2008). Fertilidad del suelo y nutrición del café en Colombia: guía práctica. Boletín Técnico Cenicafé, 32, 1-43. http://hdl.handle.net/10778/587

Sadeghian, S. (2013). Nutrición de cafetales. En Federación Nacional de Cafeteros de Colombia (Ed.), Manual del cafetero colombiano: Investigación y tecnología para la sostenibilidad de la caficultura (Vol. 2, pp. 85-116). Cenicafé.

Shoemaker, H.E., McLean, E.O., \& Pratt, P.F. (1961). Buffer methods for determination of lime requirement of soils with appreciable amount of exchangeable aluminum. Soil Science Society of America Journal, 25(4), 274-277. https://doi.org/10.2136/ sssaj1961.03615995002500040014x 
Suárez, F., \& Rodríguez, A. (1956). Aplicación de la cal en cafetos jóvenes. Revista Cafetera de Colombia, 12(129), 4294-4301. http://hdl.handle.net/10778/1108

Toro, R. (1994). Efecto de dosis crecientes de cal dolomítica en plántulas de café/Coffea arabica/var. Colombia en invernadero (Tesis de pregrado). Universidad Nacional de Colombia.

Valencia, G., \& Bravo, E. (1981). Influencia del encalamiento en la producción de cafetales establecidos. Revista Cenicafé, 32(1),3-14. http://hdl.handle.net/10778/1109 